\title{
Tubo-ovarian abscess in woman with an intrauterine device forgotten for 22 years
}

\author{
Doerte W. Luedders • Nektarios Chalvatzas • \\ Constanze Banz • Amadeus Horneman • \\ Klaus Diedrich • Andreas Kavallaris
}

Received: 7 December 2009 /Accepted: 21 December 2009/Published online: 21 January 2010

(C) Springer-Verlag 2010

\begin{abstract}
We report on a case of pelvic inflammatory disease in a 53 year old woman using an intrauterine contraceptive device (IUD) for a time period of 22 years. Clinical symptoms were fever up to $38^{\circ} \mathrm{C}$ and severe abdominal pain. Endoscopic findings were a tubo-ovarian abscess of the complete pelvis and a forgotten IUD with a smear positive for Escherichia coli and Candida albicans. The patient was treated with a surgical therapy and by antibiotic combination. Diagnosis was performed by histopathological examination.
\end{abstract}

Keywords Tubo-ovarian abscess · IUD ·

Pelvic inflammatory disease

\section{Introduction}

Tubo-ovarian abscess (TOA) is one of the major complications of acute pelvic inflammatory disease (PID). It is a polymicrobial infection typically occurring in sexually active females. [1, 2]. It occurs when microorganisms ascend from the cervix into the uterus, fallopian tubes or peritoneal cavity. Nevertheless, the development of TOA has been referred to as the end stage in the progression of

D. W. Luedders $\cdot$ N. Chalvatzas $\cdot$ C. Banz $\cdot$ A. Horneman $\cdot$

K. Diedrich $\cdot$ A. Kavallaris $(\square)$

Department of Obstetrics and Gynaecology, University Hospital of Schleswig-Holstein,

Campus Luebeck, Ratzeburger Allee 160,

23538 Luebeck, Germany

e-mail: andreas.kavallaris@uk-sh.de upper genital tract infection [3, 4]. Women in their third or fourth decade of life have predisposing factors to be affected by TOA $[4,5]$. In recent years, a new trend in the epidemiology of TOA has been observed that older women in their fifth and sixth decade of life and even in postmenopausal women suffer more frequently from TOA $[6,7]$.

We report in the following a case of acute pelvic inflammatory disease with severe TOA in a 53-year-old woman using an intrauterine contraceptive device (IUD) for a time period of 22 years.

\section{Case report}

A 53-year-old premenopausal woman was seen as an emergency case in the outpatients' clinic of the Department of Surgery. She had suffered from abdominal pain for 5 days with fever up to $38^{\circ} \mathrm{C}$ and elevated blood infection parameters. Furthermore, she suffered from obstipation and growth of abdominal girth. Due to the abdominal pain, a computed tomography was performed. It showed an unclear structure near the uterus. She was then referred to our department. The last gynaecological examination had been performed 15 years before. In our examination, an enlarged painful uterus myomatosus with an orthotropic IUD was suspected, with the IUD inserted 22 years before. The patient was of the opinion that the IUD had been lost during a menstruation long time before because her gynaecologist had been unable to locate it 15 years before.

However, the removal of the IUD initially failed in our department due to the lack of a thread. The clinical signs strongly suspected a tubo-ovarian abscess (TOA). First, a 


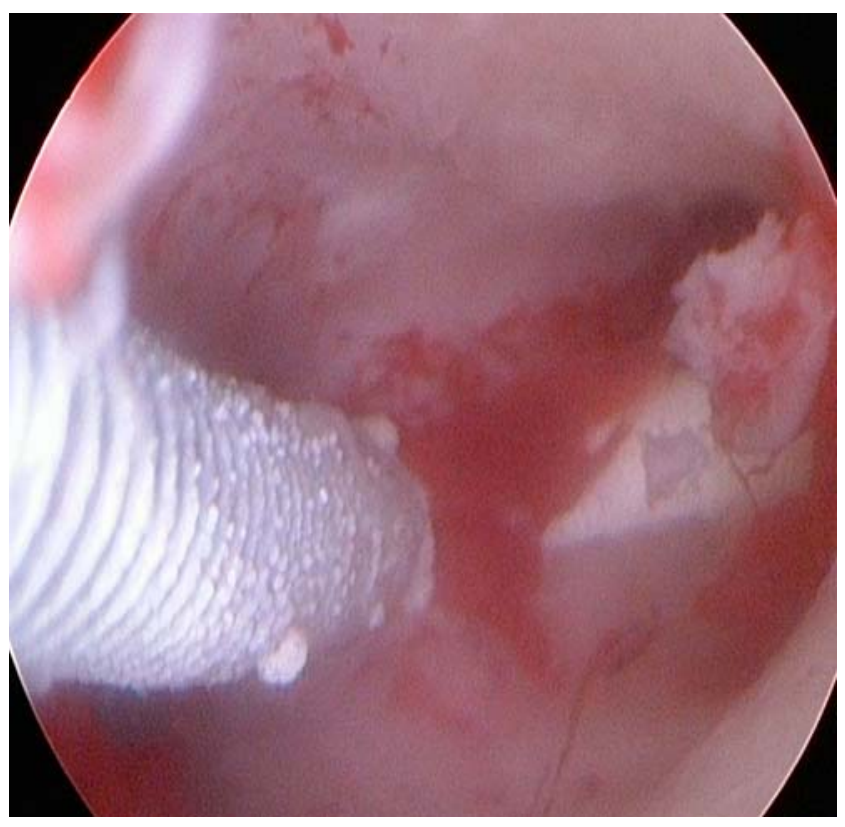

Fig. 1 IUD in situ for 22 years

conservative therapy with intravenous antibiotics was started due to a lack of sufficient fasting time then followed by surgical intervention. It showed a tubo-ovarian abscess (TOA) of the complete pelvis, an enlarged uterus myomatosus with enlarged tubes and a lost orthotopic IUD with a smear positive for Escherichia coli and Candida albicans. Diagnosis was confirmed by histopathological examination. A bacterial swab was taken intraoperatively from the

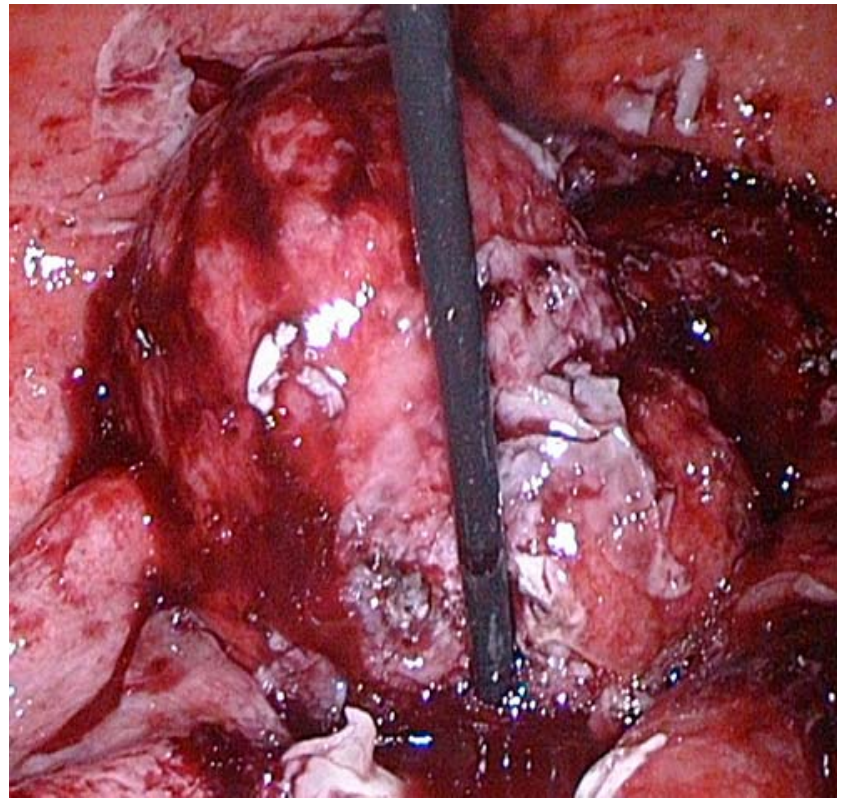

Fig. 2 Enlarged uterus myomatosus with amplified tubes and fibrin plaques

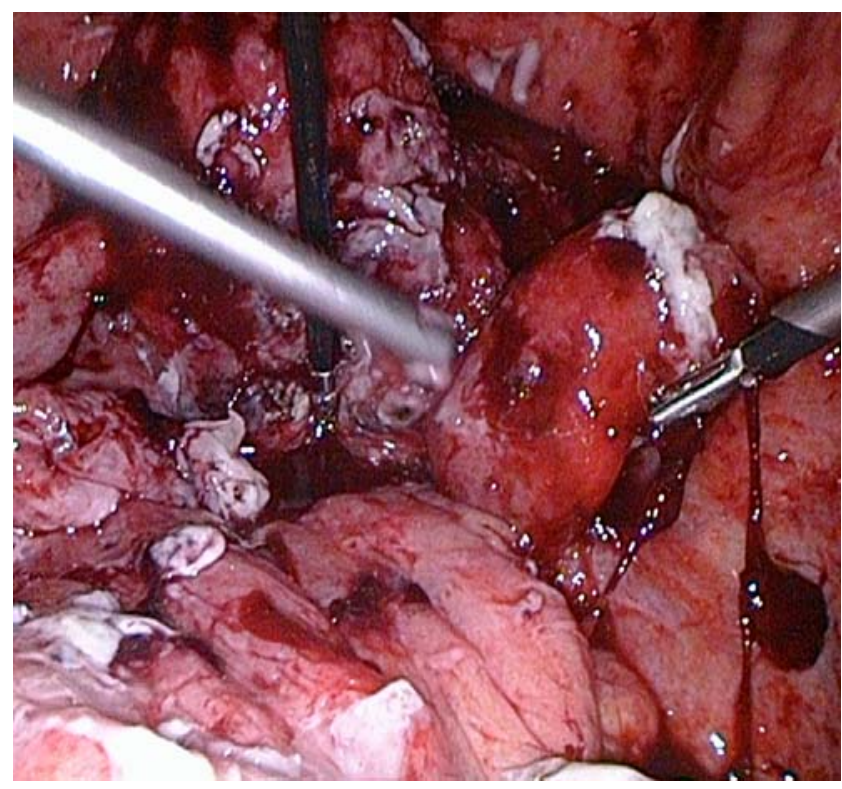

Fig. 3 Right enlarged tube with fibrin plaques

abdominal cavity. However, the microbiological examination failed to show conclusive results.

The intravenous antibiotic therapy was continued further for 5 days with a decline of all blood infection parameters.

After 1 month, a total laparoscopic hysterectomy with bilateral adnexectomy was performed in a second-look operation, showing severe adhesions within the complete pelvis. The patient was fine in a follow-up examination,

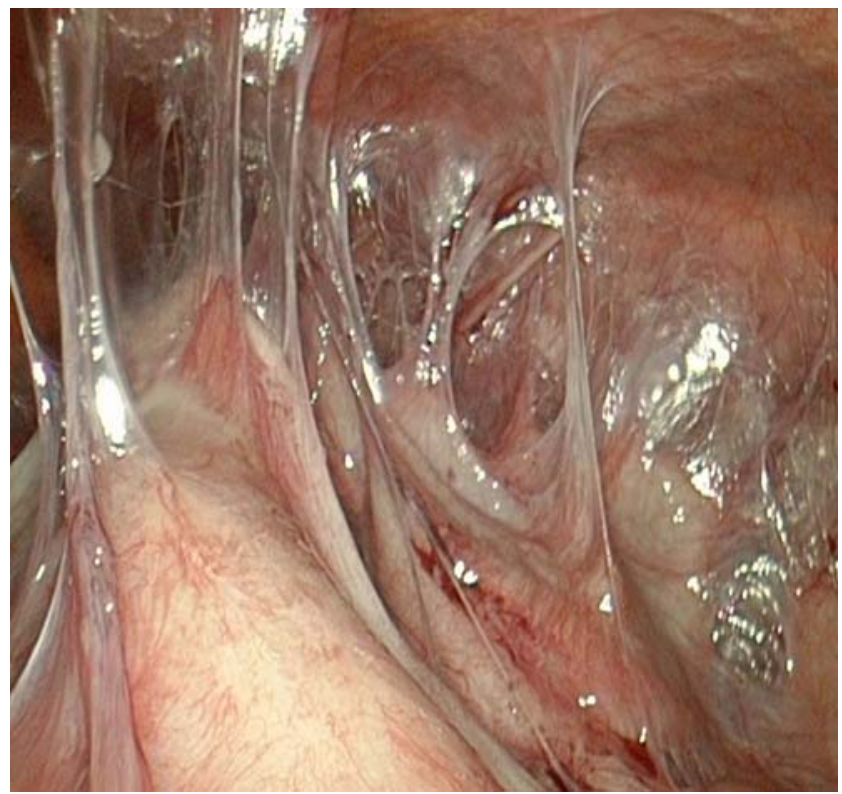

Fig. 4 One month after TOA: abdominal adhesions 


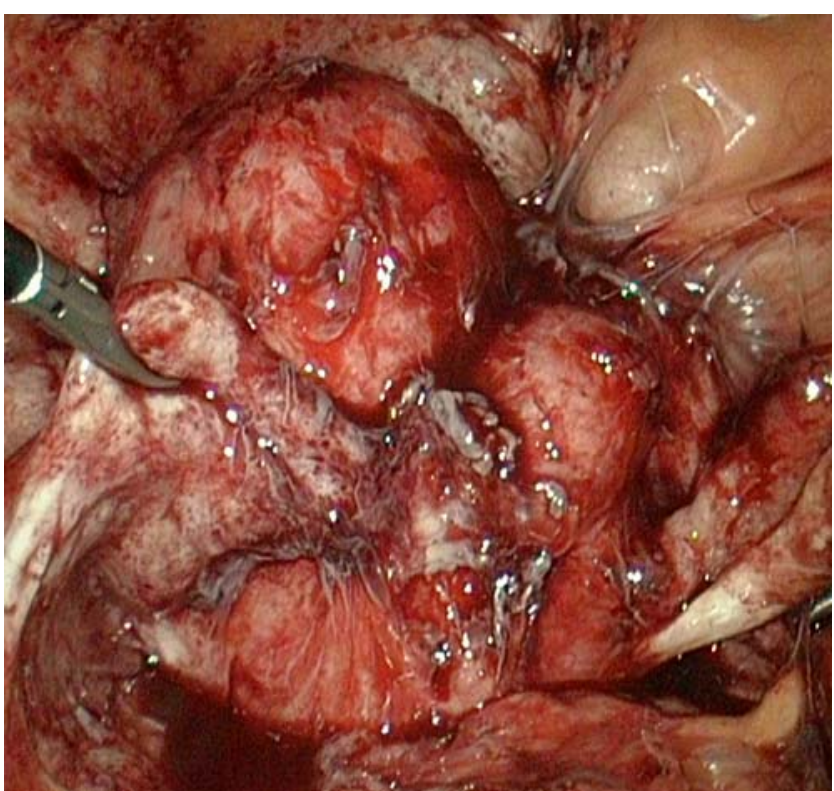

Fig. 5 Uterus myomaotosus after adhesiolysis and before total laparoscopic hysterectomy

10 weeks after total laparoscopic hysterectomy (Figs. 1, 2, $3,4,5)$.

\section{Discussion}

Tubo-ovarian abscess is one of the major complications of acute pelvic inflammatory disease. The spectrum of disease ranges from subclinical, asymptomatic infection to severe illness. Complications include chronic pelvic pain, ectopic pregnancy, tubo-ovarian abscess and infertility [1].

Clinical findings in PID supporting the diagnosis are increased cervical or vaginal discharge, elevation of infection parameters, positive bacterial smears, oral temperature of $38^{\circ} \mathrm{C}$ or greater and white blood cells on vaginal secretion.

When the diagnostic of PID is questionable, further investigation may be needed. Imaging modalities may include transvaginal ultrasound, computed tomography and magnetic resonance imaging, with transvaginal ultrasound regarded especially helpful in the diagnosis of TOA $[2,8]$.

If these imaging techniques fail, invasive examinations should be considered with laparoscopy regarded as method of choice. The procedure allows direct visualisation of all pelvic structures. Another advantage is a direct option of surgery interventions like abscess-opening or pelvic lavage and drainage.

In addition to surgery therapy, an antibiotic therapy should be considered. The antibiotic therapy should cover Neisseria gonnorrhoe, Chlamydia trachomatis and anaerobes, Gram-negative facultative bacteria and Streptococcus species [2]. The predisposing factors in the development of a TOA include nulliparity, sexual activity, previous episodes of PID, multiple sexual partners, lower socioeconomic status and the use of intrauterine devices $[6,9]$. Particularly, IUDs are associated with an increased risk for ascending infections [9] with high absolute numbers, especially as they are considered worldwide as one of the most preferred methods of reversible contraception [10].

These findings are not in line with the results of Franklin et al., describing those older women may also have predisposing factors to suffer from TOA. They showed that the mean age of all 60 patients was $>40$ years. The authors thus concluded a new trend in the epidemiology of TOA, indicating a more aggressive compared to younger women $[6,7]$. One suggestion is that previous asymptomatic or subclinical infections may progress silently to the TOA stage over a long period of time. Another possible explanation of the seemingly higher incidence of TOA in older women is the low index of suspicion among physicians dealing with abdominal pain in older age women. Although specific single causes of pelvic inflammatory disease are seldom identified, cultures from a TOA usually show a polymicrobial infection, especially with bacteria such as E. coli, Bacteroides fragilis, or other anaerobes (Kim). However, especially in cases of rarer pathogens, results from a bacterial culture of the abdominal cavity may lead to a more specific treatment [11], as patients with lost IUDs may suffer more often from other than commonly sexually transmitted infections

Another risk factor for the development to TOA in older women may be a significantly prolonged use of PID, seen more frequently in older patients with definitive wish for contraception. This assumption is supported by the publication of Charonis and Larsson, indicating that in women around the age of 40 who have a IUD $>5$ years have a significantly higher risk to suffer from get TOA than women with an IUD in situ for less than 5 years.

That is in line with our case report dealing with a 53-year-old woman using an intrauterine device for a period of 22 years. The last gynaecological examination had been performed 15 years before.

The constellation of older women with a history of a hypothetically lost IUD, abdominal pain and other clinical signs of infections should attention gynaecologists and other physicians to include TOA in their differential diagnosis and avoid delay of adequate treatment. The latter may also included surgical interventions to lance abscesses and extract forgotten IUDs. 


\section{References}

1. Banikarim C, Chacko MR (2005) Pelvic inflammatory disease in adolescents. Semin Pediatr Infect Dis 16:175-180

2. Crossman SH (2006) The challenge of pelvic inflammatory disease. Am Fam Physician 73:859-864

3. Benigno BB (1981) Medical and surgical management of the pelvic abscess. Clin Obstet Gynecol 24:1187-1197

4. Franklin EW 3rd, Hevron JE Jr, Thompson JD (1973) Management of the pelvic abscess. Clin Obstet Gynecol 16:66-79

5. Granberg S, Gjelland K, Ekerhovd E (2009) The management of pelvic abscess. Best Pract Res Clin Obstet Gynaecol 23:667-678

6. Halperin R, Levinson O, Yaron M, Bukovsky I, Schneider D (2003) Tubo-ovarian abscess in older women: is the woman's age a risk factor for failed response to conservative treatment? Gynecol Obstet Invest 55:211-215

7. Kamprath S, Merker A, Kühne-Heid R, Schneider A (1997) Abdominal actinomycosis with IUD. Zentralbl Gynakol 119:21-24

8. Krivak TC, Cooksey C, Propst AM (2004) Tubo-ovarian abscess: diagnosis, medical and surgical management. Compr Ther 30:93-100

9. Scott WC (1978) Pelvic abscess in association with intrauterine contraceptive device. Am J Obstet Gynecol 131:149-156

10. Charonis G, Larsson PG (2009) Prolonged use of intrauterine contraceptive device as a risk factor for tubo-ovarian abscess. Acta Obstet Gynecol Scand 88:680-684

11. Kim SH, Kim SH, Yang DM, Kim KA (2004) Unusual causes of tubo-ovarian abscess: CT and MR imaging findings. Radiographics 24:1575-1589 